\title{
Machine Coordinate Values Exchange Model Design and Analysis*
}

\author{
Ming-Fu LIN** and Kao-Hua CHANG*** \\ ** Center for General Education, National Kaohsiung University of Applied Science \\ Chien Kung Campus 415 Chien Kung Road, Kaohsiung 807, Taiwan, R.O.C. \\ E-mail: mingfu@cc.kuas.edu.tw \\ *** Department of Mold and Die Engineering, National Kaohsiung University of Applied Science \\ Chien Kung Campus 415 Chien Kung Road, Kaohsiung 807, Taiwan, R.O.C.
}

\begin{abstract}
Continuing improvements in computer and machine tool technology have led to many new and fascinating applications in $\mathrm{CAD} / \mathrm{CAM}$ systems. One of the most fruitful areas of $\mathrm{CNC}$ machine research is kinematic analysis, which describes the mapping between the set of cutter position and orientation and the set of joint variables. There has thus far been relatively little research into the mapping between the set of joint variables of machine tool and set of joint variables of another machine tool. In this thesis we develop a method called machine coordinate values exchange (MCVE) model, which aims to deal with this kind of analysis. This proposed methodology is a mathematical form, applicable to both academic research and production, and enables the direct and rapid generation of joint variables. This study also presents a novel graph, called an isometric schematic, to conceptualize the prototype of multi-axis machine, and establishes and shows the feasibility of the MCVE model for two different types of machine tools.
\end{abstract}

Key words: MCVE Model, Isometric Schematic, Multi-Axis Machine, Kinematic Analysis, $\mathrm{CNC}$

\section{Introduction}

Kinematic analysis consists of direct kinematic and inverse kinematic analysis. As regards direct kinematic analysis, the cutter position and orientation are computed once the joint variables are known. Inverse kinematic analysis consists of determination of the joint variables corresponding to a given cutter position and orientation. Compared with the direct kinematic analysis, the inverse kinematic analysis is more practical and extensively used.

Computer Numerical Control (CNC) refers specifically to a computer "controller" that reads NC-code and drives a machine tool, which is a powered mechanical device typically used to fabricate metal components by the selective removal of metal. A kinematic model of the machine is essential, as it must convert the position and orientation of a cutting tool, represented by the cutter location (CL) data and the tool axis vectors, into machine coordinates of a tool path for the $\mathrm{CNC}$ machine controller. This conversion is commonly referred to as post-processing.

A number of studies over the past several decades have focus on the conceptual design of planner mechanisms ${ }^{(1) \sim(3)}$. However, design methodologies for the structural synthesis of open-type mechanisms with special motions were not available until Chen and Yan $^{(4)}$ proposed a methodology for synthesizing the mechanisms of machining centers with required topology and motion characteristics. The work of Yan and $\mathrm{Kuo}^{(5)}$ is devoted to the study of topological characteristics of mechanisms with variable topologies, considering the 
configuration singularity. Research on the configuration of machine tool has been mounting steadily in the past few decades, after Reshetov and Portman ${ }^{(6)}$ proposed the arrangement code concept for synthesizing the machine tool configuration with the same shaping function. Another intriguing study is that of Ito and Shinno, who established a design methodology for the modular construction system of machine tools, or a generating method for structural configuration. This approach synthesizes each possible type of machine tool, in which a machine tool as a whole and modular complex is represented with the directed $\operatorname{graph}^{(7)}$.

Note that the machine tool mechanism's output motion is specified and described with respect to a Cartesian coordinate system, called the set of cutter position and orientation. The motion parameter of a one degree of freedom joint is typically represented by a variable that describes the relative position between the two parts (links). All the joint variables in a mechanism form a vector of joint coordinates that define a space commonly called a set of joint variables. Therefore, machine tool kinematic analysis is simply the study of mapping between the set of cutter position and orientation and the set of joint variables. Mapping from the set of joint variables to the set of cutter position and orientation is called direct kinematics, while the reverse mapping is called inverse kinematics. However, no studies investigate the mapping between the set of joint variables of two different types of machine tools.

A machine tool can be treated as the combination of a set of serially connected parts. In practice, almost all five-axis machine tools have three slip joints and two rotary joints ${ }^{(8)}$. Motion transformation between any pair of rigid bodies generally involves six parameters. There is a simple method that only requires four motion parameters to represent the relative posture between two consecutive parts. This method (representation), which is well know and extensively used, was introduced by Denavit and Hartenberg (D-H) ${ }^{(9)}$. Tutunea-Fatan, Feng, She, and Lee develop a generic kinematic model to analysis five-axis machine tool in order to determine the slip and rotary joint movements ${ }^{(10),(11)}$. Most studies that use this representation to investigate multi-axis machining have commonly identified the need for an isometric sketch, a parameter table, and a model to analyze the kinematic structure of the machine. Hsieh and Lin present a kinematic model to produce multi-flute drills on a six-axis $\mathrm{CNC}$ tool-grinding machine. The ability matrix of a six-axis $\mathrm{CNC}$ tool-grinding machine is established by the D-H notation ${ }^{(12)}$. It is worth noting that isometric sketch of a multi-axis machine tool is usually complex, and may easily confuse the reader.

In view of the preceding introduction, the two major purposes of this study are as follows: (1) to develop a novel diagram that may lead to a better understanding of multi-axis machine tool configuration; (2) to establish a new transformation model from the set of joint variables of one machine to that of another machine.

\section{Isometric schematic and ability matrix of milling machine tool}

As mentioned above, a machine tool can be treated as a kinematic chain (the combination of a set of serially connected parts). In more scholarly words an ability matrix can describe the kinematic model of a machine tool. This section uses the following framework to derive the ability matrix of milling machine.

\subsection{Isometric schematic of machine tool}

Isometric sketch or schematic is very useful for kinematic analysis of robot or machine tool since Denavit introduced D-H notation. For example, the fig. 1 shows the isometric sketch and schematic of the slider-rotor-rotor mechanism that forms a sub-structural pattern (main flow of force) of machine tool. The column is the fixed part, which is the frame of reference for all the moving components, as well as links (sliding block, spindle head and 
spindle), and joints.

Since link of machine tool has only one degree of freedom in relative motion. According to the research of Reshetov and Portman, the arrangement code of this mechanism is a four-digit number each digit of which acquires a value from 0 to 6 . In relative motion, the translational motion of the column relative to the sliding block along axis Z. Symbol 3 corresponds to this motion. Analogously, the sliding block rotates around axis $Z$, in a direction opposite to the direction of rotation of the spindle head, i.e., symbol = 6 , and, finally, spindle head rotates around axis Y, i.e., symbol $=5$. Since the column is always assumed stationary, then a zero is added to the left of the code. Thus, the arrangement code of this mechanism is 0365 .

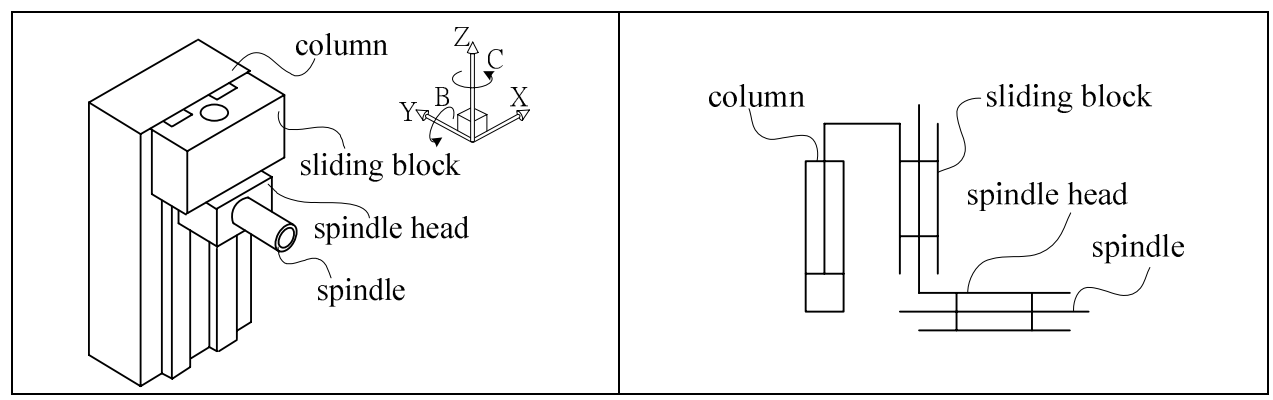

Fig. 1 Comparison of isometric sketch (left) and schematic (right) styles of a slider-rotor-rotor (0365) mechanism diagrams.
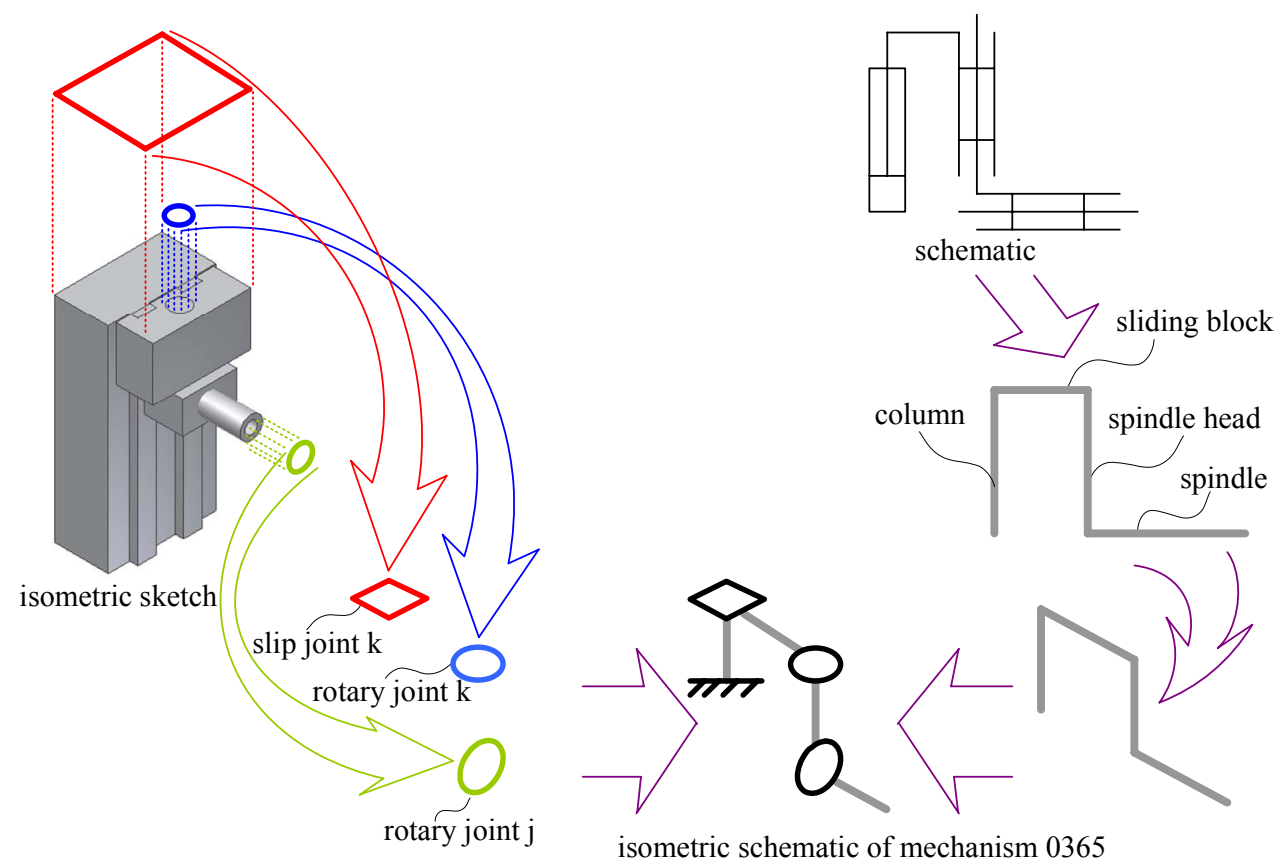

Fig. 2 An isometric schematic is a three dimension kinematic diagram of the mechanical system that shows the topological structure.

A mechanism is a heart of a machine. The mechanism of CNC machine consists of parts (links) and primary joints. Figure 2 shows a novel diagram, called an isometric schematic, of this kinematic chain. An isometric view of an object can be obtained by choosing the viewing direction in a way that the angles between the projection of the $\mathrm{x}, \mathrm{y}$, and $\mathrm{z}$ axes (denote by $\mathrm{i}, \mathrm{j}$, and $\mathrm{k}$ ) are all the same, or $120^{\circ}$. In an isometric schematic, parts are denoted by gray edges, slip joints by rhombus vertices, and rotary joints by ellipse vertices. New representations of rotary joints, slip joints, and parts are listed in Table 1. 
Table 1 Isometric schematic symbols

rotary joints

\subsection{Ability matrix of the vertical milling machine}

It is very hard to understand the whole concept of kinematic chain based on the isometric sketch of a multiple-axis machine tool. A graphic (Fig. 3) example may help see this point more clearly.

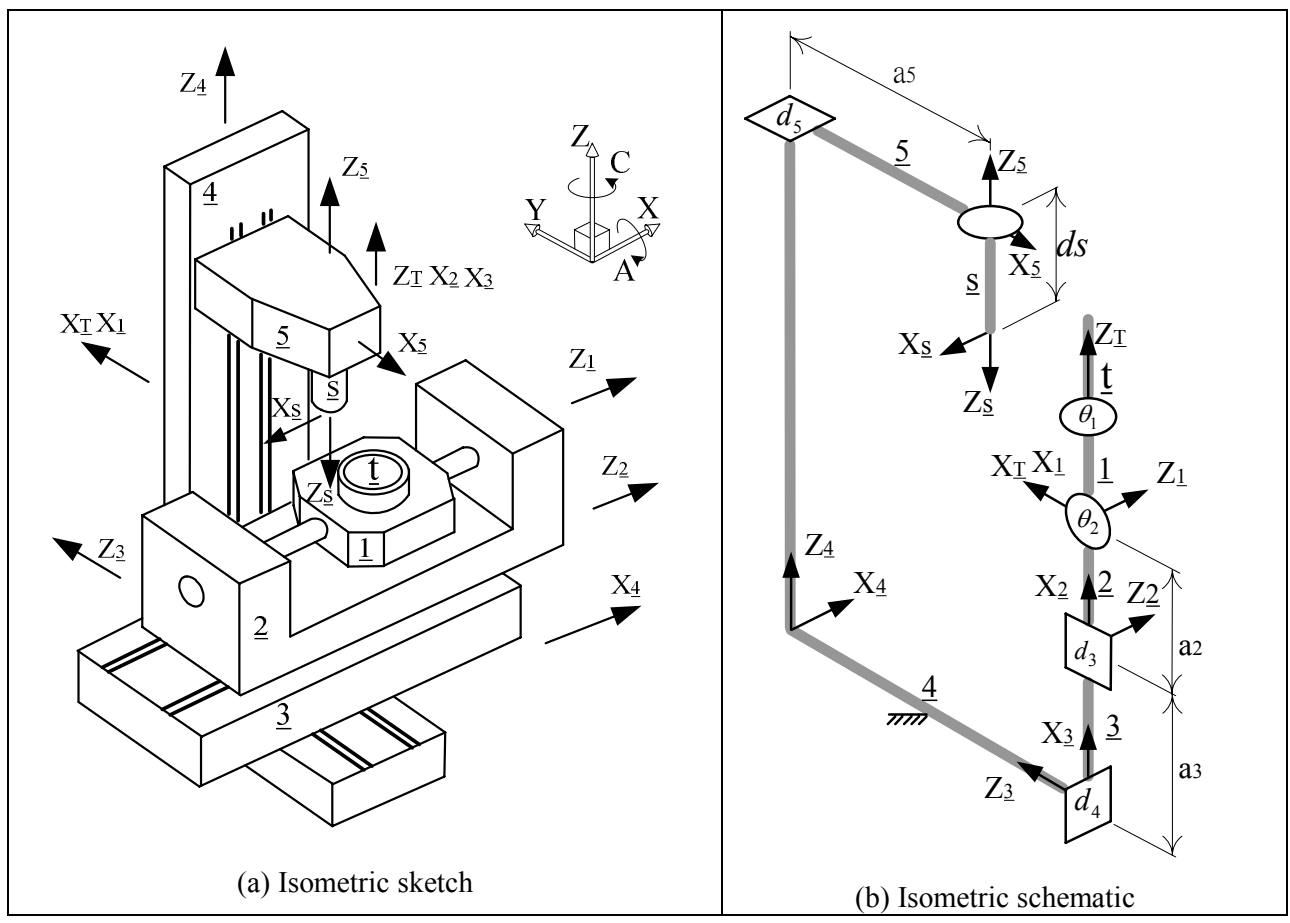

Fig. 3 Vertical milling machine 641203

Figure 3 shows a part-frame assignment example of a vertical milling machine 641203 . Since this machine consist of many parts, some part frames cannot be observable directly. Unlike the isometric sketch (Fig. 3a), the isometric schematic (Fig. 3b) that introduce the relevant parameters used in the model makes it very easy to understand the whole concept of kinematic chain. Note that $\theta_{1}, \theta_{2}, d_{3}, d_{4}$ and $d_{5}$, which fill in the blank of rotary and slip symbols, are joint variables. Nothing fills in the blank of the spindle rotary symbol, because functional redundancy will occur in a vertical milling task since the spindle (cutting tool) rotation about the approach direction is irrelevant to completion of the task.

Once the frames $\langle\mathbf{X Y Z}\rangle_{i}(i=\underline{t}, \underline{1}, \underline{2}, \underline{3}, \underline{4}, \underline{5}$, and $\underline{s})$ have been assigned according to D-H notation, the displacement from the frame of one part to that of the next part may be represented by a matrix dependent on the four parameters $\theta_{\mathrm{i}}, d_{\mathrm{i}}, \mathrm{a}_{\mathrm{i}}$, and $\alpha_{\mathrm{i}}$. This defines the relative position of the systems, as specified in Table 2. Thus, the matrix of the displacement from $\langle\mathbf{X Y Z}\rangle_{i-1}$ (fixed in part $\left.i-1\right)$ to $\langle\mathbf{X Y Z}\rangle_{i}$ (fixed in part $i$ ) is 


$$
{ }^{i-1} \mathbf{A}_{i}=\operatorname{Rot}\left(\mathbf{Z}, \theta_{i}\right) \operatorname{Tra}\left(0,0, d_{i}\right) \operatorname{Tra}\left(\mathrm{a}_{i}, 0,0\right) \operatorname{Rot}\left(\mathbf{X}, \alpha_{i}\right)
$$

The D-H convention make it possible to construct the kinematic function by combining the individual coordination transformations expressed in Eq. (1) into a homogeneous transformation matrix:

Table 2 D-H parameters of the vertical milling machine 641203

\begin{tabular}{ccccccc}
\hline Link & $\underline{1}$ & $\underline{2}$ & $\underline{3}$ & $\underline{4}$ & $\underline{5}$ & $\underline{s}$ \\
\hline$\theta_{i}$ & $\theta_{1}$ & $\theta_{2}$ & 0 & $90^{\circ}$ & $-90^{\circ}$ & $-90^{\circ}$ \\
$d_{i}$ & 0 & 0 & $d_{3}$ & $d_{4}$ & $d_{5}$ & $-d_{s}$ \\
$\mathrm{a}_{\mathrm{i}}$ & 0 & $-\mathrm{a}_{2}$ & $-\mathrm{a}_{3}$ & 0 & $\mathrm{a}_{5}$ & 0 \\
$\alpha_{i}$ & $90^{\circ}$ & 0 & $90^{\circ}$ & $90^{\circ}$ & 0 & $180^{\circ}$ \\
\hline
\end{tabular}

$$
\begin{aligned}
& { }^{t} \mathbf{T}_{\underline{s}}={ }^{t} \mathbf{A}_{\underline{1}}{ }^{1} \mathbf{A}_{\underline{2}}{ }^{2} \mathbf{A}_{\underline{3}}{ }^{3} \mathbf{A}_{\underline{4}}{ }^{4} \mathbf{A}_{\underline{5}}{ }^{5} \mathbf{A}_{\underline{s}}= \\
& {\left[\begin{array}{cccc}
-S \theta_{1} & C \theta_{1} S \theta_{2} & -C \theta_{1} C \theta_{2} & \left(-a_{2}-a_{3}+d_{5}-d_{s}\right) C \theta_{1} C \theta_{2}+\left(d_{4}-a_{5}\right) C \theta_{1} S \theta_{2}+d_{3} S \theta_{1} \\
C \theta_{1} & S \theta_{1} S \theta_{2} & C \theta_{2} S \theta_{1} & \left(-a_{2}-a_{3}+d_{5}-d_{s}\right) C \theta_{2} S \theta_{1}+\left(d_{4}-a_{5}\right) S \theta_{1} S \theta_{2}-d_{3} C \theta_{1} \\
0 & -C \theta_{2} & -S \theta_{2} & \left(a_{5}-d_{4}\right) C \theta_{2}-\left(a_{2}+a_{3}-d_{5}+d_{s}\right) S \theta_{2} \\
0 & 0 & 0 & 1
\end{array}\right]}
\end{aligned}
$$

where $C \theta=\cos \theta, S \theta=\sin \theta$ etc. The matrix of the displacement from $\langle\mathbf{X Y Z}\rangle_{\underline{w}}$ (fixed in workpiece) to $\langle\mathbf{X Y Z}\rangle_{\underline{\underline{t}}}$ (fixed in table) is

$$
{ }^{\underline{w}} \mathbf{A}_{\underline{t}}=\left[\begin{array}{cccc}
C\left(90^{\circ}\right) & -S\left(90^{\circ}\right) & 0 & 0 \\
S\left(90^{\circ}\right) & C\left(90^{\circ}\right) & 0 & 0 \\
0 & 0 & 1 & d_{t} \\
0 & 0 & 0 & 1
\end{array}\right]=\left[\begin{array}{cccc}
1 & -1 & 0 & 0 \\
1 & 1 & 0 & 0 \\
0 & 0 & 1 & d_{t} \\
0 & 0 & 0 & 1
\end{array}\right]
$$

where $d_{\mathrm{t}}$ is the offset between the origins of the frames $\langle\mathbf{X Y Z}\rangle_{\underline{w}}$ and $\langle\mathbf{X Y Z}\rangle_{\underline{t}}$. The matrix of the displacement from $\langle\mathbf{X Y Z}\rangle_{\underline{\underline{S}}}$ (fixed in spindle) to $\langle\mathbf{X Y Z}\rangle_{\underline{\underline{c}}}$ (fixed in cutting tool) is

$$
{ }^{\underline{s}} \mathbf{A}_{\underline{c}}=\left[\begin{array}{cccc}
1 & 0 & 0 & 0 \\
0 & 1 & 0 & 0 \\
0 & 0 & 1 & d_{c} \\
0 & 0 & 0 & 1
\end{array}\right]
$$

where $d_{\mathrm{s}}$ and $d_{\mathrm{c}}$ are the offset values between the origins of the frames $\langle\mathbf{X Y Z}\rangle_{s}$ and $\langle\mathbf{X Y Z}\rangle_{\underline{c}}$. The matrix product of Eqs. $(2) \sim(4)$ is the ability matrix of the vertical milling machine. 


$$
{ }^{\underline{w}} \mathbf{T}_{\underline{c}}={ }^{w} \mathbf{A}_{\underline{t}}{ }_{\underline{t}} \mathbf{T}_{\underline{s}}{ }^{\underline{s}} \mathbf{A}_{\underline{c}}=\left[\begin{array}{ccc}
-C \theta_{1}-S \theta_{1} & C \theta_{1} S \theta_{2}-S \theta_{1} S \theta_{2} & -C \theta_{1} C \theta_{2}+\mathrm{C} \theta_{2} S \theta_{1} \\
C \theta_{1}-S \theta_{1} & C \theta_{1} S \theta_{2}+S \theta_{1} S \theta_{2} & -C \theta_{1} C \theta_{2}-C \theta_{2} S \theta_{1} \\
0 & -C \theta_{2} & -S \theta_{2} \\
0 & 0 & 0
\end{array}\right.
$$

$$
\left.\begin{array}{c}
\left(a_{2}+a_{3}-d_{5}+d_{c}+d_{s}\right) C \theta_{2}\left(S \theta_{1}-C \theta_{1}\right)+\left(d_{4}-a_{5}\right) S \theta_{2}\left(C \theta_{1}-S \theta_{1}\right)+d_{3}\left(S \theta_{1}+C \theta_{1}\right) \\
\left(-a_{2}-a_{3}+d_{5}-d_{c}-d_{s}\right) C \theta_{2}\left(S \theta_{1}+C \theta_{1}\right)+\left(d_{4}-a_{5}\right) S \theta_{2}\left(S \theta_{1}+C \theta_{1}\right)+d_{3}\left(S \theta_{1}-C \theta_{1}\right) \\
\left(a_{5}-d_{4}\right) C \theta_{2}+\left(-a_{2}-a_{3}+d_{5}-d_{c}-d_{s}\right) S \theta_{2}+d_{t}
\end{array}\right]
$$

\section{Inverse kinematics of the vertical milling machine 641203}

Before solving the joint variables of the vertical milling machine, it is necessary to define the relative position and orientation of the cutting tool frame with respect to the workpiece frame (Fig. 4). Let ${ }^{\underline{w}} \mathbf{X}_{\underline{c}},{ }^{\underline{w}} \mathbf{Y}_{\underline{c}},{ }^{\underline{w}} \mathbf{Z}_{\underline{c}}$ and ${ }^{\underline{w}} \mathbf{P}_{\underline{c}}$ be the unit vectors of the tool frame axes and the position of the tool tip with respect to the workpiece frame, respectively. The coordinate transformation from the workpiece frame to the cutting tool frame can be written in terms of a $(4 \times 4)$ matrix:

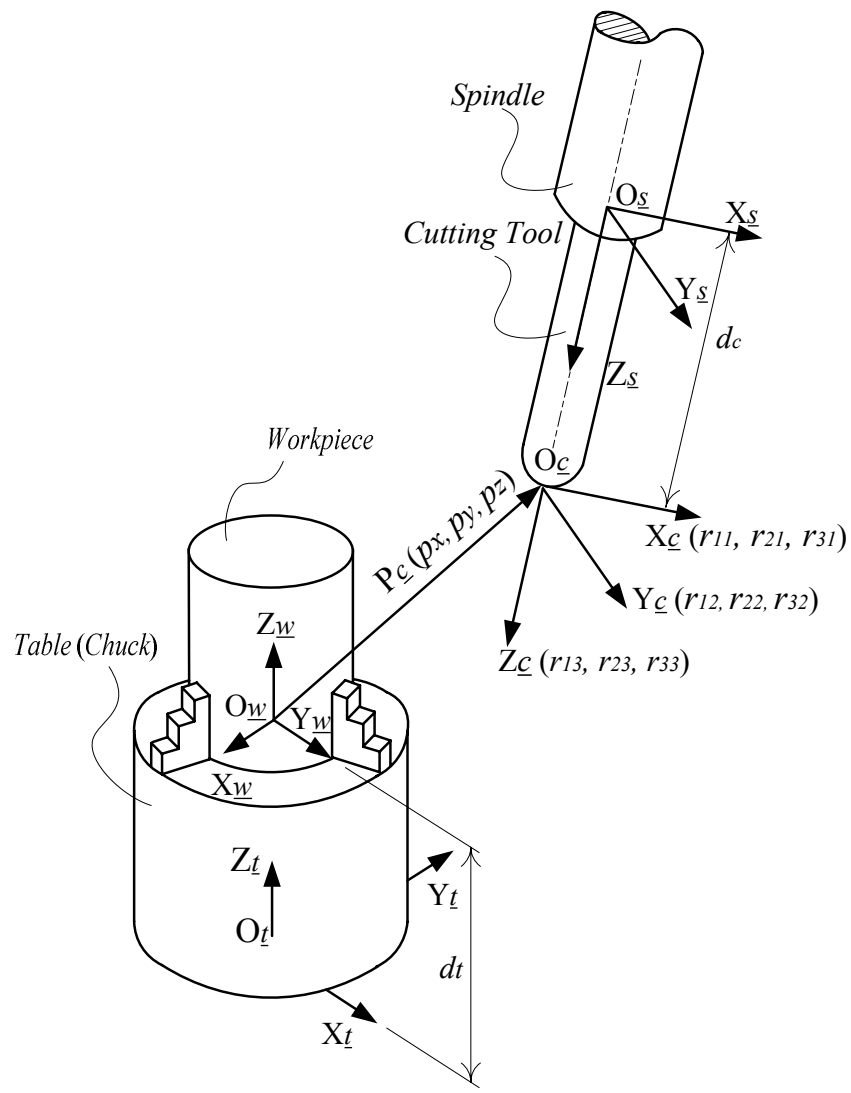

Fig. 4 Relative position and orientation of the cutting tool frame with respect to the workpiece frame

$$
{ }^{\underline{w}} \mathbf{M}_{\underline{c}}=\left[\begin{array}{cccc}
{ }^{\underline{w}} \mathbf{X}_{\underline{c}} & { }^{\underline{w}} \mathbf{Y}_{\underline{c}} & { }^{\underline{w}} \mathbf{Z}_{c} & { }^{w} \mathbf{P}_{c} \\
0 & 0 & 0 & 1
\end{array}\right]=\left[\begin{array}{cccc}
r_{11} & r_{12} & r_{13} & p_{x} \\
r_{21} & r_{22} & r_{23} & p_{y} \\
r_{31} & r_{32} & r_{33} & p_{z} \\
0 & 0 & 0 & 1
\end{array}\right]
$$


The inverse kinematics analysis consists of determining the joint variables that correspond to a given position and orientation of the cutting tool. In the case of the vertical milling machine, it is necessary to convert the given data (CL data and orientation of the tool) into machine coordinates, which control the motions of the machine. Equation (5) shows the ability matrix of the vertical milling machine. Theoretically, the desired five joint variables $\left(\theta_{1}, \theta_{2}, d_{3}, d_{4}\right.$ and $\left.d_{5}\right)$ can be determined by equating the elements of Eqs. (5) and (6).

$$
{ }^{\underline{w}} \mathbf{M}_{\underline{c}}={ }^{\underline{w}} \mathbf{T}_{\underline{c}}
$$

Note that the joint variables are mixed together, since they are all on the right sight of the equation, and the matrix equation is difficult to solve. To overcome this difficulty, multiply both sides of the equation by $\left[{ }^{t} \mathbf{A}_{\underline{1}}\right]^{-1}\left[{ }^{\underline{w}} \mathbf{A}_{\underline{t}}\right]^{-1}$, giving

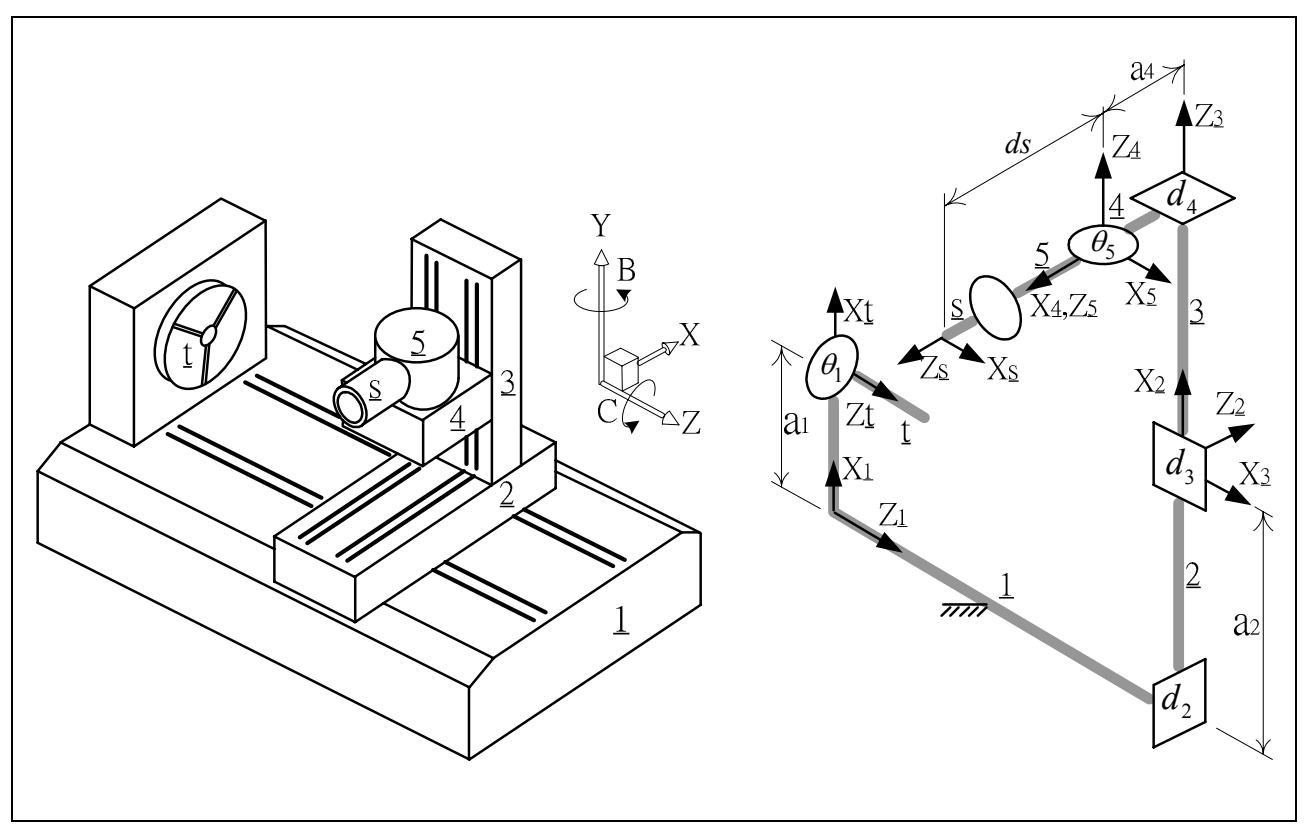

Fig. 5 The universal turning center 603215 .

$$
\begin{aligned}
& {\left[{ }^{t} \mathbf{A}_{\underline{1}}\right]^{-1}\left[{ }^{\underline{w}} \mathbf{A}_{\underline{t}}\right]^{-1}{ }^{\underline{w}} \mathbf{M}_{\underline{c}}={ }^{-1} \mathbf{A}_{\underline{2}}{ }^{2} \mathbf{A}_{\underline{3}}{ }^{3} \mathbf{A}_{\underline{4}}{ }^{4} \mathbf{A}_{\underline{5}}{ }^{\underline{5}} \mathbf{T}_{\underline{c}}} \\
& {\left[\begin{array}{cccc}
r_{21} C \theta_{1}-r_{11} S \theta_{1} & r_{22} C \theta_{1}-r_{12} S \theta_{1} & r_{23} C \theta_{1}-r_{13} S \theta_{1} & p_{y} C \theta_{1}-p_{x} S \theta_{1} \\
r_{31} & r_{32} & r_{33} & -d_{t}+p_{z} \\
r_{11} C \theta_{1}+r_{21} S \theta_{1} & r_{12} C \theta_{1}+r_{22} S \theta_{1} & r_{13} C \theta_{1}+r_{23} S \theta_{1} & p_{x} C \theta_{1}-p_{y} S \theta_{1} \\
0 & 0 & 0 & 1
\end{array}\right]=} \\
& {\left[\begin{array}{cccc}
0 & S \theta_{2} & -C \theta_{2} & \left(-a_{2}-a_{3}+d_{5}-d_{s}-d_{c}\right) C \theta_{2}+\left(d_{4}-a_{5}\right) S \theta_{2} \\
0 & -C \theta_{2} & -S \theta_{2} & \left(-a_{2}-a_{3}+d_{5}-d_{s}-d_{c}\right) S \theta_{2}+\left(-d_{4}+a_{5}\right) C \theta_{2} \\
-1 & 0 & 0 & d_{3} \\
0 & 0 & 0 & 1
\end{array}\right]} \\
& \theta_{1}=2 \tan ^{-1}\left(\frac{-r_{11} \pm \sqrt{r_{11}^{2}+r_{21}^{2}}}{r_{21}}\right)
\end{aligned}
$$




$$
\begin{aligned}
& \theta_{2}=S^{-1}\left(-r_{33}\right) \\
& d_{3}=p_{x} C \theta_{1}-p_{y} S \theta_{1} \\
& d_{4}=a_{5}+\left(d_{t}-p_{z}\right) C \theta_{2}+p_{y} C \theta_{1} S \theta_{2}-p_{x} S \theta_{1} S \theta_{2} \\
& d_{5}=a_{2}+a_{3}+d_{c}+d_{s}+p_{y} C \theta_{1} C \theta_{2}-p_{x} S \theta_{1} C \theta_{2}+\left(p_{z}-d_{t}\right) S \theta_{2} C \theta_{2}
\end{aligned}
$$

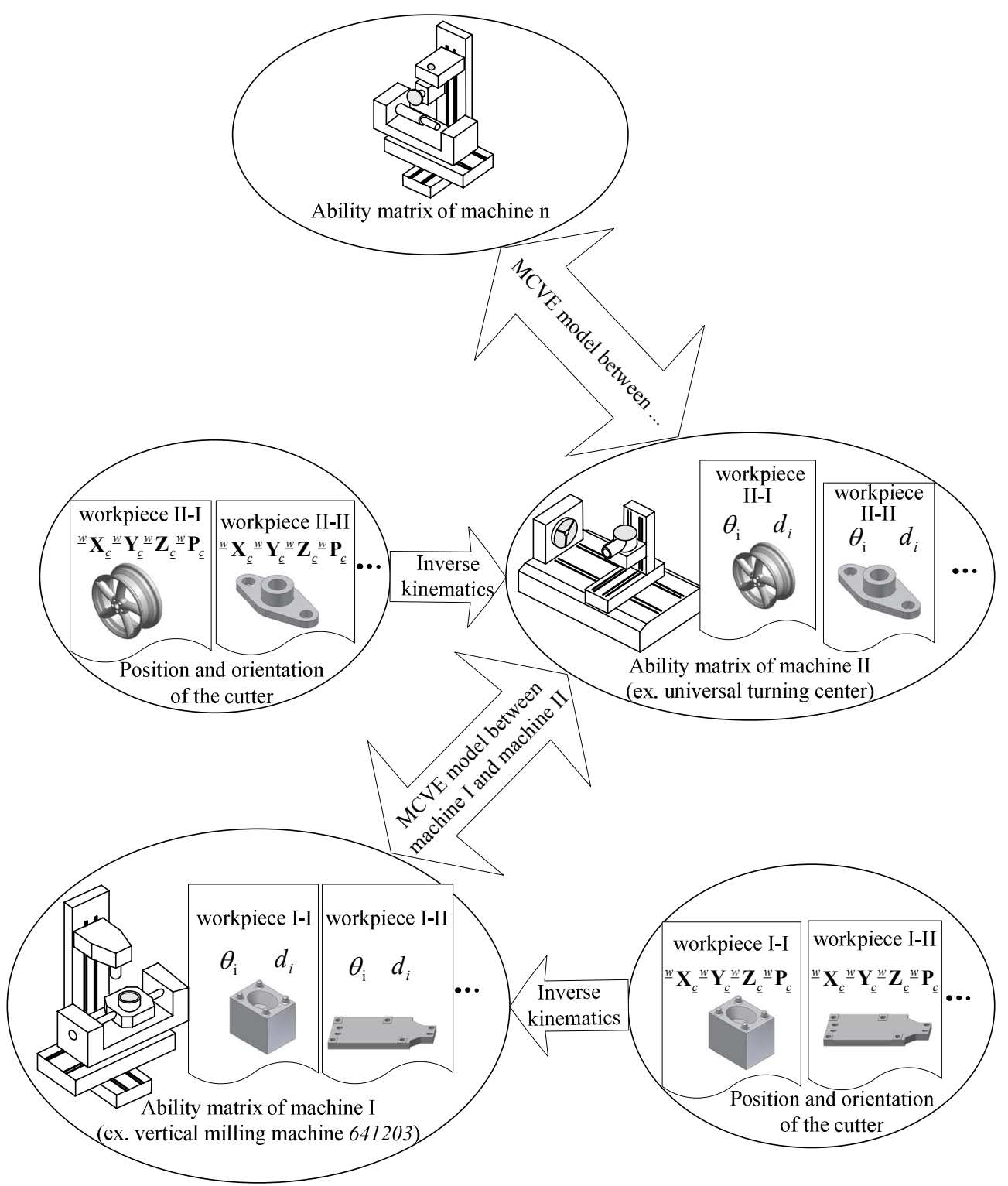

Fig. 6 An illustration of inverse kinematics mapping and MCVE model between two machines.

\section{MCVE model of the universal turning center 603125}

This section uses a novel method (called MCVE model) to solve the joint variables of the universal turning center (Fig. 5) instead of inverse kinematic analysis.

The main object of an MCVE model is to conduct inter-machine kinematics analysis for the multi-axis machines by employing a mapping formula to relate the dimension (distance 
and angular) words of one machine and the dimension words of another machine. It is of paramount importance that this method can exchange a massive amount of dimension words. If the relationship between the set of joint variables of the vertical milling machine and that of the universal turning center exhibit a one-to-one correspondence, then the mapping from the set of joint variables of the vertical milling machine to that of the universal turning center is effective (Fig. 6).

Table 3 specifies the D-H parameters of the universal turning center 603125 and its ability matrix can be expressed as

Table 3 D-H parameters of the universal turning center 603125

\begin{tabular}{ccccccc}
\hline Link & $\underline{1}$ & $\underline{2}$ & $\underline{3}$ & $\underline{4}$ & $\underline{5}$ & $\underline{s}$ \\
\hline$\theta_{i}$ & $\theta_{1}$ & 0 & $90^{\circ}$ & $-90^{\circ}$ & $\theta_{5}$ & 0 \\
$d_{i}$ & 0 & $d_{2}$ & $d_{3}$ & $d_{4}$ & 0 & $d_{\mathrm{s}}$ \\
$\mathrm{a}_{\mathrm{i}}$ & $-\mathrm{a}_{1}$ & $\mathrm{a}_{2}$ & 0 & $\mathrm{a}_{4}$ & 0 & 0 \\
$\alpha_{i}$ & 0 & $90^{\circ}$ & $90^{\circ}$ & 0 & $-90^{\circ}$ & 0 \\
\hline
\end{tabular}

$$
\begin{aligned}
& { }^{t} \mathbf{T}_{\underline{s}}=\left[\begin{array}{cccc}
-C \theta_{5}^{\prime} S \theta_{1}^{\prime} & C \theta_{1}^{\prime} & -S \theta_{1}^{\prime} S \theta_{5}^{\prime} & \left(-a_{1}+a_{2}+d_{4}\right) C \theta_{1}^{\prime}-\left(a_{4}-d_{3}+d_{s} S \theta_{5}^{\prime}\right) S \theta_{1}^{\prime} \\
C \theta_{1}^{\prime} C \theta_{5}^{\prime} & S \theta_{1}^{\prime} & C \theta_{1}^{\prime} S \theta_{5}^{\prime} & \left(-a_{1}+a_{2}+d_{4}\right) S \theta_{1}^{\prime}+\left(a_{4}-d_{3}+d_{s} S \theta_{5}^{\prime}\right) C \theta_{1}^{\prime} \\
S \theta_{5}^{\prime} & 0 & -C \theta_{5}^{\prime} & d_{2}-d_{s} C \theta_{5}^{\prime} \\
0 & 0 & 0 & 1
\end{array}\right] \\
& { }^{\underline{w}} \mathbf{A}_{\underline{t}}=\left[\begin{array}{cccc}
1 & -1 & 0 & 0 \\
1 & 1 & 0 & 0 \\
0 & 0 & 1 & d_{t}^{\prime} \\
0 & 0 & 0 & 1
\end{array}\right] \\
& { }^{s} \mathbf{A}_{\underline{c}}=\left[\begin{array}{cccc}
1 & 0 & 0 & 0 \\
0 & 1 & 0 & 0 \\
0 & 0 & 1 & d_{c}^{\prime} \\
0 & 0 & 0 & 1
\end{array}\right] \\
& { }^{\underline{w}} \mathbf{T}_{\underline{c}}={ }^{\underline{w}} \mathbf{A}_{\underline{t}}{ }^{t} \mathbf{T}_{\underline{s}}{ }^{\underline{s}} \mathbf{A}_{\underline{c}}= \\
& {\left[\begin{array}{cccc}
-C \theta_{1}^{\prime} C \theta_{5}^{\prime} & -S \theta_{1}^{\prime} & -C \theta_{1}^{\prime} C \theta_{5}^{\prime} & \left(a_{1}^{\prime}-a_{2}^{\prime}-d_{4}^{\prime}\right) S \theta_{1}^{\prime}-\left(a_{4}^{\prime}-d_{3}^{\prime}+d_{s}^{\prime} S \theta_{5}^{\prime}+d_{c}^{\prime} S \theta_{5}^{\prime}\right) C \theta_{1}^{\prime} \\
-C \theta_{5}^{\prime} S \theta_{1}^{\prime} & C \theta_{1}^{\prime} & -S \theta_{1}^{\prime} S \theta_{5}^{\prime} & \left(-a_{1}^{\prime}+a_{2}^{\prime}+d_{4}^{\prime}\right) C \theta_{1}^{\prime}-\left(a_{4}^{\prime}-d_{3}^{\prime}+d_{s}^{\prime} S \theta_{5}^{\prime}+d_{c}^{\prime} S \theta_{5}^{\prime}\right) S \theta_{1}^{\prime} \\
S \theta_{5}^{\prime} & 0 & -C \theta_{5}^{\prime} & d_{2}^{\prime}+d_{t}^{\prime}-d_{s}^{\prime} C \theta_{5}^{\prime}-d_{c}^{\prime} C \theta_{5}^{\prime} \\
0 & 0 & 0 & 1
\end{array}\right]}
\end{aligned}
$$

Solve for the five joint variables of the universal turning center 603125 by equating the corresponding elements of the above matrix (Eq. 17) with the ability matrix of the vertical milling machine 641203 .

$$
\theta_{1}^{\prime}=S^{-1}\left(C \theta_{1} S \theta_{2}-S \theta_{1} S \theta_{2}\right)
$$




$$
\begin{aligned}
& \theta_{5}^{\prime}=C^{-1}\left(S \theta_{2}\right) \\
& d_{2}^{\prime}=\left(d_{s}^{\prime}+d_{c}^{\prime}\right) C \theta_{5}^{\prime}+\left(a_{5}-d_{4}\right) C \theta_{2}+\left(-a_{2}-a_{3}+d_{5}-d_{c}-d_{s}\right) S \theta_{2}+d_{t}-d_{t}^{\prime} \\
& d_{3}^{\prime}=c_{2}+c_{3} C \theta_{1}^{\prime}+c_{4} S \theta_{1}^{\prime} \\
& d_{4}^{\prime}=c_{1}+c_{4} C \theta_{1}^{\prime}-c_{3} S \theta_{1}^{\prime}
\end{aligned}
$$

where $c_{1}=\left(a_{1}^{\prime}-a_{2}^{\prime}\right), \quad c_{2}=\left(a_{4}^{\prime}+d_{s}^{\prime} S \theta_{5}^{\prime}+d_{c}^{\prime} S \theta_{5}^{\prime}\right)$,

$$
\begin{aligned}
& c_{3}=\left(a_{2}+a_{3}-d_{5}+d_{c}+d_{s}\right) C \theta_{2}\left(S \theta_{1}-C \theta_{1}\right)+\left(d_{4}-a_{5}\right) S \theta_{2}\left(C \theta_{1}-S \theta_{1}\right)+d_{3}\left(S \theta_{1}+C \theta_{1}\right), \\
& c_{4}=\left(d_{5}-a_{2}-a_{3}-d_{c}-d_{s}\right) C \theta_{2}\left(S \theta_{1}+C \theta_{1}\right)+\left(d_{4}-a_{5}\right) S \theta_{2}\left(S \theta_{1}+C \theta_{1}\right)+d_{3}\left(S \theta_{1}-C \theta_{1}\right) .
\end{aligned}
$$

\section{Simulation}

A side wall axial relief feed (swarf) operation is a typical five-axis cutting operation. This cutting strategy uses the side of the cutting tool to machine tilted walls. This classic five-axis strategy is suitable for a wide variety of pattern geometries, and allows the toolpath to contain several steps along the walls. The tool's $4^{\text {th }}$ and $5^{\text {th }}$ axis positions are automatically determined from the selected walls as the tool moves around the pattern.

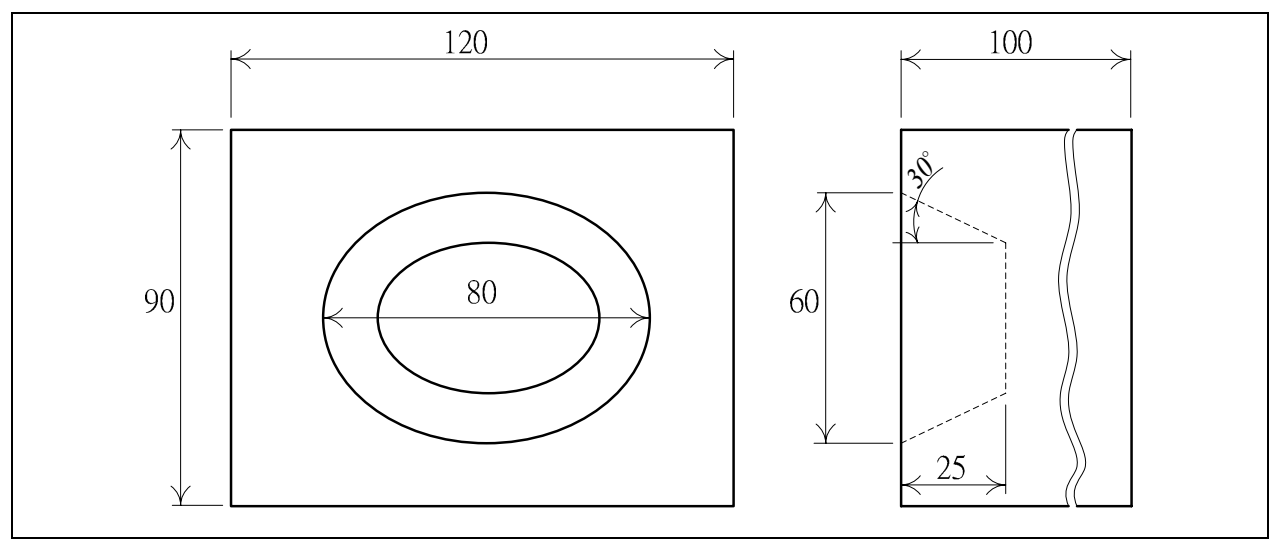

Fig. 7 The top view and the side view of this simulation workpiece.

Figure 7 illustrates the top view and the side view of this simulation workpiece, where the designed tilted wall is an elliptic ruled surface.

In this case, the joint variables $\theta_{1}, \theta_{2}, d_{3}, d_{4}$, and $d_{5}$ of the vertical milling machine are rotational around (or translational along) the $\mathrm{C}, \mathrm{A}, \mathrm{X}, \mathrm{Y}$, and $\mathrm{Z}$ axes. The joint variables of the universal turning center are $\theta_{1}, \theta_{5}, d_{2}, d_{3}$, and $d_{4}$, and are rotational around (or translational along) the $\mathrm{C}, \mathrm{B}, \mathrm{Z}, \mathrm{X}$, and $\mathrm{Y}$ axes, respectively. The toolpath for this pattern is generated on the basis of theoretical values according to Eqs. (8) (13) and Eqs. (18) (22), as shown in Fig. 8.

To demonstrate the validity of the proposed methodology, the programs which were generated on the basis of theoretical values were simulated using the EdgeCAM software. The cutting tool used for the simulations is a flat end mill with a long flute length that has a 10-mm diameter. Figs. 9 and 10 show the toolpath simulations and machine simulations of the vertical milling machine and the universal turning center. The CAM simulation results 
of the vertical milling machine are compatible with the results of the universal turning center.
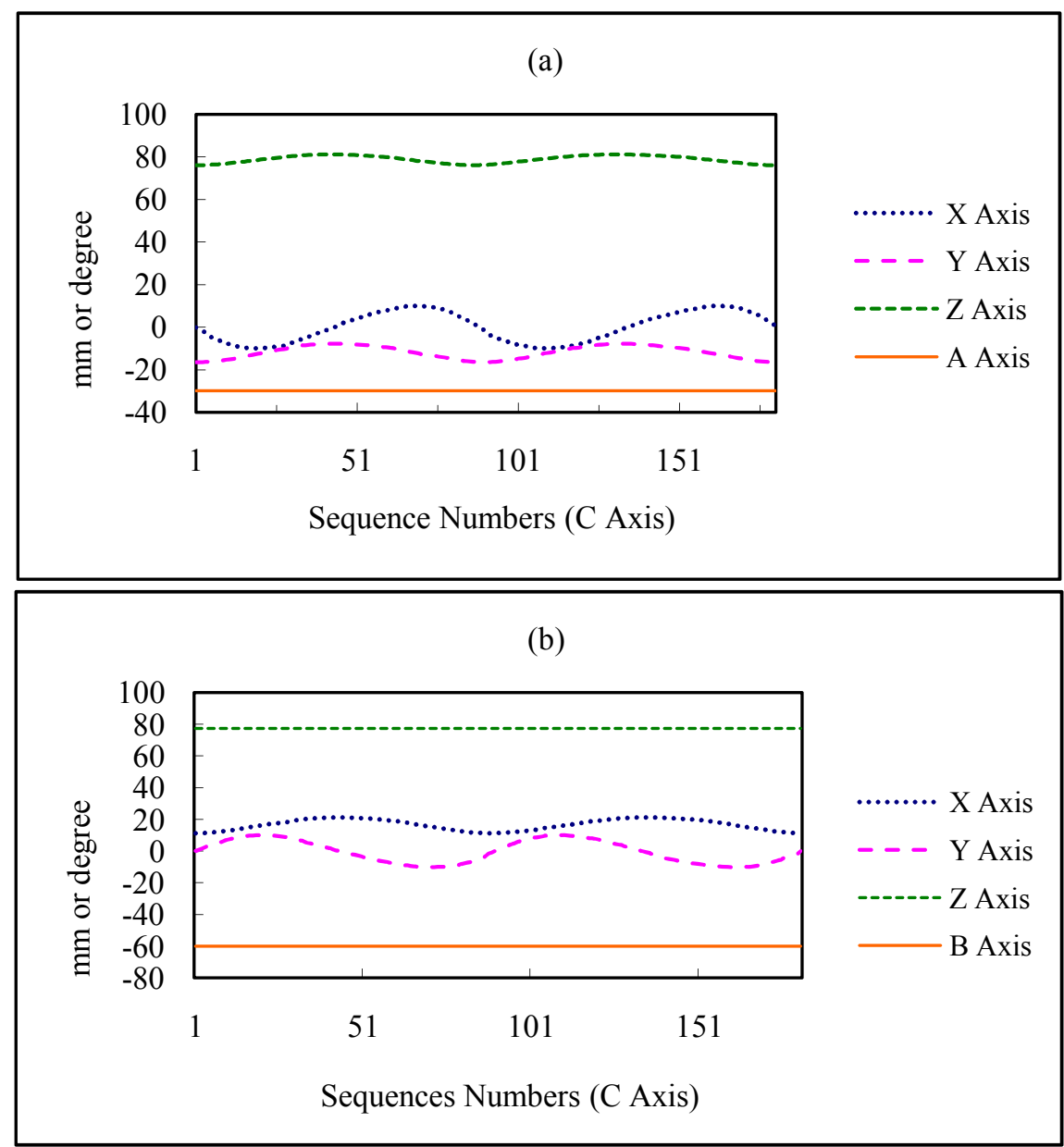

Fig. 8 Coordinates of the tool (for the simulations) position and orientation of (a) vertical milling machine 641203 and (b) universal turning center 603125 .

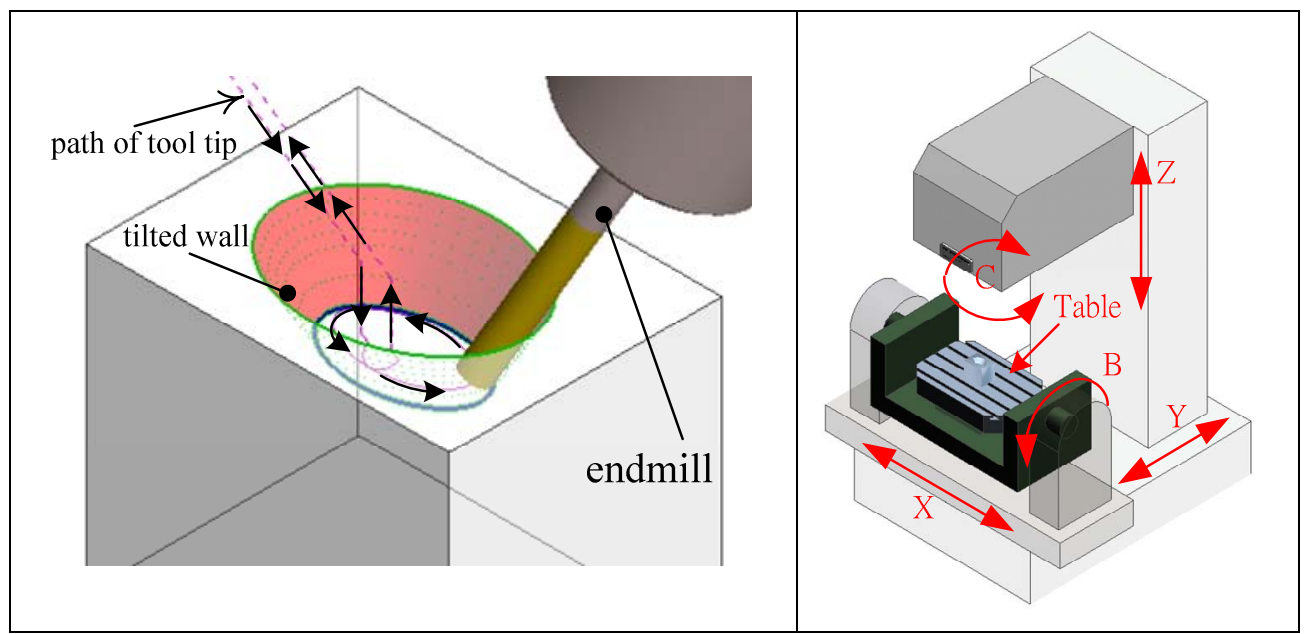

Fig. 9 Toolpath simulations (left) and machine simulations (right) of the vertical milling machine 641203 . 


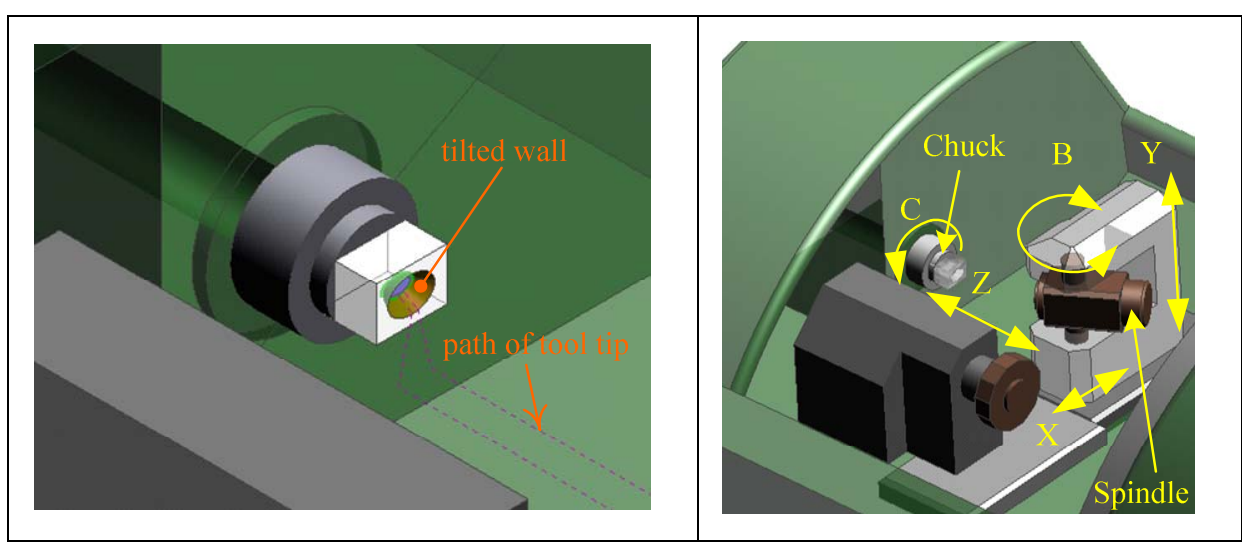

Fig. 10 Toolpath simulations (left) and machine simulations (right) of the universal turning center 603215 .

\section{Conclusion}

This study has shown the development of a MCVE model which yields the kinematic equations for the universal turning machine. As anticipated, the MCVE model can be applied to multi-axis machining. The results of the MCVE model are fairly consistent with what we know about the result of inverse kinematic analysis.

CNC program is generally in block of four sections. These four sections are as follows: sequence numbers, preparatory function, dimensions, and miscellaneous function. The proposed MCVE model is a mathematical method used to exchange the dimension words between different types of machine tools. While this research is still at the initial stages, our findings will have broad implications in areas of CNC machine research. The model described here has the potential to serve as the basis for studies into methods that exchange complete programs between different types of machine tools.

\section{Acknowledges}

The authors are thankful to the National Science Council of Taiwan for supporting this research under grant NSC- NSC99-2221-E-151-019 and Enago (www.enago.tw) for the English language review.

\section{References}

( 1 ) Bushsbaum, F., Freudenstein, F., Synthesis of kinematic structure of geared kinematic chains and other mechanisms, Journal of Mechanisms, No.5(1970), pp. 357-392.

( 2 ) Freudenstein, F., Maki, E. E., The creation of mechanisms according to kinematic structure and function, Journal of Environmental and Planning B, No. 6(1979), pp. 357-391.

( 3 ) Erdman, A. G., Nelson, E., Peterson, J., Bowen, J., Type and dimensional synthesis of casement window mechanisms, ASME, (1980), paper no. 80-DET-78.

( 4 ) Chen, F. C., Yan, H. S., Configuration synthesis of machining centres with tool change mechanisms, International Journal of Machine Tools and Manufacture, No.39(1999) pp. 273-295.

( 5) Yan, H.S., Kuo, C. H., Topological representation and characteristics of variable kinematic joints, ASME Transactions, Journal of Mechanical Design, Vol. 128, No .2(2006), pp.384-391.

( 6 ) Reshetov, D. N., Portman, V. T., Accuracy of Machine Tools, (1988), ASME Press, 
New York.

( 7 ) Shinno, H., Ito, Y., Computer Aided Concept Design for Structural Configuration of Machine Tools: Variant Design Using Directed Graph, Journal of Mechanisms, Transmission, and Automation in Design, Transactions of the ASME, Journal mech. des., No.109(1987), pp. 372-376.

( 8 ) Bohez, E. L. J., Five-axis milling machine tool kinematic chain design and analysis, in-ternational Journal of Machine Tools and Manufacture, Vol. 42, No. 4(2002), pp. 505-520.

( 9 ) Denavit, J., Hartenberg, R. S., A kinematic notation for lower pair mechanisms based on matrices, ASME Journal of Applied Mechanics, Vol. 22 , No.2(1955), pp. 215-221.

(10) She, C. H., Lee, R. S., A Postprocessor Based on the Kinematics Model for General Five-Axis Machine Tools, Journal of Manufacturing Processes, Vol. 2, No. 2 (2000), pp. 131-141.

(11 ) Tutunea-Fatan, O. R., Feng, H. Y., Configurations analysis of five-axis machine tools using a generic kinematic model, International Journal of Machine Tools and Manufacture. No. 44(2004), pp. 1235-1243.

(12 ) Hsieh, J. F., Lin, P. D., Production of multifluted drill on six-axis CNC tool-grinding machine, I. J. of machine tools and manufacture, 43(2003), pp. 1117-1127. 